\title{
Stretching and Breaking of Ultrathin $\mathrm{MoS}_{2}$
}

\author{
Simone Bertolazzi, ${ }^{\dagger}$ Jacopo Brivio, ${ }^{\dagger}$ and Andras Kis* \\ Electrical Engineering Institute, Ecole Polytechnique Federale de Lausanne (EPFL), CH-1015 Lausanne, Switzerland. ${ }^{\dagger}$ These authors contributed equally to this work.
}

$\mathrm{T}$ wo-dimensional crystals, consisting of single or few atomic layers extracted from layered materials such as graphite or $\mathrm{MoS}_{2}{ }^{1-3}$ are attracting a great deal of interest due to their promising potential for applications in nanotechnology. Graphene is the best known 2D material because of its high mobility, presence of massless Dirac fermions, ${ }^{4,5}$ and a wealth of interesting physical phenomena such as the fractional quantum Hall effect. ${ }^{6}$ Other $2 \mathrm{D}$ materials such as transition metal dichalcogenides or BN could have practical applications and fundamental properties complementary to those of graphene, although they are at this point much less explored.

Single-layer $\mathrm{MoS}_{2}$ is a typical two-dimensional semiconductor from the class of layered transition metal dichalcogenides (TMD). Individual layers, $6.5 \AA$ thick, can be extracted from bulk crystals using the micromechanical cleavage technique commonly associated with the production of graphene, $^{2,3}$ lithium-based intercalation, ${ }^{7,8}$ or liquid phase exfoliation ${ }^{9}$ and used as ready-made blocks for electronic device fabrication. ${ }^{10}$ Bulk $\mathrm{MoS}_{2}$ is an indirect gap semiconductor with a band gap of $1.2 \mathrm{eV}$ (ref 11). Reducing the number of layers modifies the band structure and, as a consequence, monolayer $\mathrm{MoS}_{2}$ becomes a direct gap semiconductor ${ }^{12-15}$ with a band gap of $1.8 \mathrm{eV}$ (ref 14) due to quantum confinement. ${ }^{15}$ The presence of a band gap in monolayer $\mathrm{MoS}_{2}$ makes it interesting for applications in nanoelectronics where it allows the fabrication of transistors with low power dissipation and current on/off ratios exceeding $10^{8}$ at room temperature. Together with the possibility of large-scale liquid-based processing of $\mathrm{MoS}_{2}$ and related $2 \mathrm{D}$ materials, ${ }^{9} \mathrm{MoS}_{2}$ could also be very interesting for applications in flexible electronics where it would combine high performance with low cost. It is however not clear at this point if monolayer $\mathrm{MoS}_{2}$ would be characterized by mechanical properties

\section{ABSTRACT}

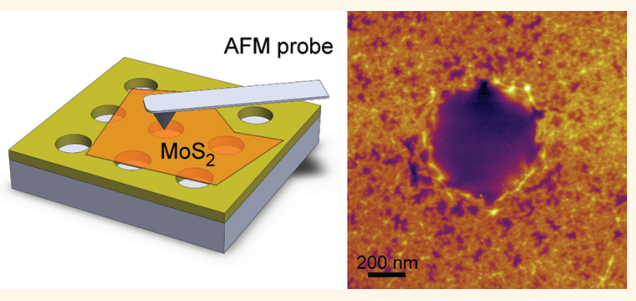

We report on measurements of the stiffness and breaking strength of monolayer $\mathrm{MoS}_{2}$, a new semiconducting analogue of graphene. Single and bilayer $\mathrm{MoS}_{2}$ is exfoliated from bulk and transferred to a substrate containing an array of microfabricated circular holes. The resulting suspended, free-standing membranes are deformed and eventually broken using an atomic force microscope. We find that the in-plane stiffness of monolayer $\mathrm{MoS}_{2}$ is $180 \pm 60 \mathrm{Nm}^{-1}$, corresponding to an effective Young's modulus of $270 \pm 100 \mathrm{GPa}$, which is comparable to that of steel. Breaking occurs at an effective strain between 6 and $11 \%$ with the average breaking strength of $15 \pm 3 \mathrm{Nm}^{-1}$ (23 $\mathrm{GPa}$ ). The strength of strongest monolayer membranes is $11 \%$ of its Young's modulus, corresponding to the upper theoretical limit which indicates that the material can be highly crystalline and almost defect-free. Our results show that monolayer $\mathrm{MoS}_{2}$ could be suitable for a variety of applications such as reinforcing elements in composites and for fabrication of flexible electronic devices.

KEYWORDS: two-dimensional materials · dichalcogenides $\cdot \mathrm{MoS}_{2} \cdot \mathrm{AFM} \cdot$ mechanical properties

necessary for integration with stretchable polymer substrates in order to produce high-end bendable electronics.

Previous measurements on $\mathrm{MoS}_{2}$ and $W_{2}$ nanotubes, ${ }^{16,17}$ which can be thought of as monolayers of $\mathrm{MoS}_{2}$ and $\mathrm{WS}_{2}$ wrapped up in the form of a cylinder, show superior mechanical properties with Young's modulus reaching $255 \mathrm{GPa}$ and strength reaching $11 \%$ of its Young's modulus. ${ }^{18,19}$ Subnanometer $\mathrm{MoS}_{2}$ nanowires on the other hand have lower Young's modulus, $120 \mathrm{GPa}^{20}$

Here, we report on the measurement of the in-plane elastic modulus and breaking strength of single and bilayer $\mathrm{MoS}_{2}$. MoS 2 consists of a stack of covalently bonded S-Mo-S layers weakly interacting with each other via van der Waals forces. The in-plane *Address correspondence to
andras.kis@epfl.ch.

Received for review August 19, 2011 and accepted November 9, 2011.

Published online

$10.1021 / \mathrm{nn} 203879 f$

(C) XXXX American Chemical Society 
crystal structure is determined by polar covalent bonds resulting from the overlap between the $4 d$ and $3 p$ electron orbitals of molybdenum and sulfur. The inplane stiffness $\left(E^{2 \mathrm{D}}\right)$ of an ideal defect-free single-layer $\mathrm{MoS}_{2}$ is related to the effective spring constant of these molecular bonds. A defect-free material would have the upper theoretical limit of the breaking strength ( $\sigma_{\max }^{2 \mathrm{D}} \approx 10 \%$ of the Young's modulus). ${ }^{21}$ We use nanoindentation in an atomic force microscope in order to perform nanomechanical measurements on ultrathin $\mathrm{MoS}_{2}$ suspended over circular holes in patterned substrates, using a technique previously used for measurements on multilayer ${ }^{22,23}$ and single-layer graphene. ${ }^{24}$ Mechanical properties of graphene have also been probed by performing uniaxial measurements. ${ }^{25,26}$

\section{RESULTS AND DISCUSSION}

Single- and few-layer $\mathrm{MoS}_{2}$ was extracted from bulk crystals of naturally occurring $\mathrm{MoS}_{2}$ using the micromechanical cleavage technique commonly employed for the production of graphene. ${ }^{1}$ We found that direct exfoliation on patterned substrates yielded a relatively small number of samples, presumably because of reduced adhesion between the substrate and $\mathrm{MoS}_{2}$. We therefore employed a transfer technique ${ }^{27}$ to first exfoliate $\mathrm{MoS}_{2}$ on polymer films and then transfer the resulting material onto prepatterned surfaces. $\mathrm{MoS}_{2}$ is first deposited on Si substrates covered with $270 \mathrm{~nm}$ $\mathrm{SiO}_{2}$ on top of which polyvinyl alcohol (PVA) and polymethyl methacrylate (PMMA) films have previously been spin-coated. We have reported elsewhere ${ }^{28}$ that this $\mathrm{SiO}_{2}$ thickness results in optimal visibility of monolayer $\mathrm{MoS}_{2}$. Once an interesting flake with an optical contrast corresponding to a monolayer is located using an optical microscope, AFM is used to verify the thickness. The sample is then immersed in water to dissolve the PVA film and release the PMMA layer which ends up floating on the water surface. The film is moved onto a pierced glass slide and aligned to the new substrate with the help of a micromanipulator. The new substrate consisted of a $270 \mathrm{~nm}$ thick $\mathrm{SiO}_{2}$ (ref 28) patterned with $550 \mathrm{~nm}$ diameter holes defined by e-beam lithography followed by dry etching. Finally, the sample is kept in vacuum at $400^{\circ} \mathrm{C}$ for $4 \mathrm{~h}$ in order to release the polymer film without the use of solvents that could break $\mathrm{MoS}_{2}$ during drying. In this way, we obtain a relatively high yield of intact $\mathrm{MoS}_{2}$ membranes suspended over circular holes. Figure 1a shows a monolayer flake after the transfer on the new substrate, while Figure $1 \mathrm{~b}$ shows a corresponding AFM image of the sample topography. Resulting suspended ultrathin layers of $\mathrm{MoS}_{2}$ are tightly clamped to the edges of the holes, without visible wrinkles or discontinuities.

Mechanical properties of the membranes were probed with indentation experiments ${ }^{29}$ using an AFM (Asylum Research Cypher) with a standard silicon
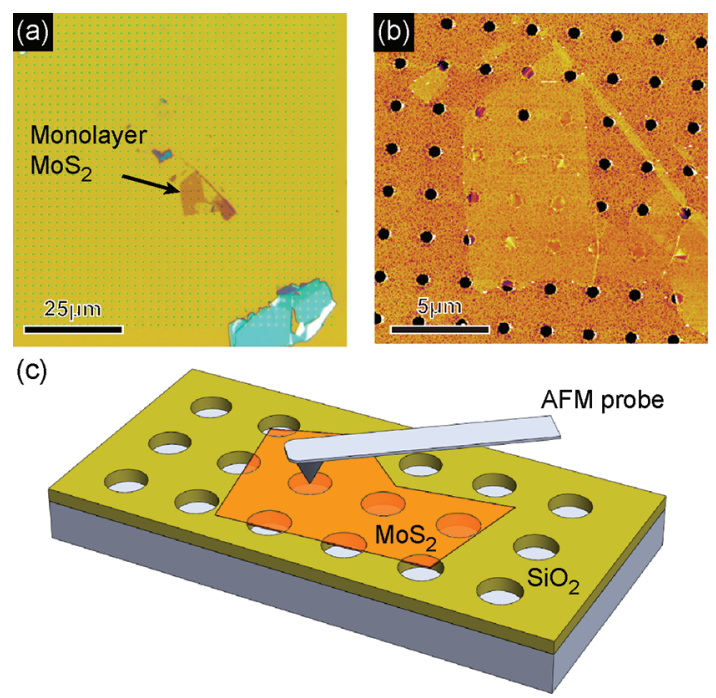

Figure 1. Sample preparation and the measurement method. (a) Optical image of a monolayer $\mathrm{MoS}_{2}$ flake transferred onto the prepatterned $\mathrm{SiO}_{2}$ substrate containing an array of circular holes $550 \mathrm{~nm}$ in diameter. (b) AFM image of the same single-layer $\mathrm{MoS}_{2}$ as in part (a) shows that the monolayer is clean, free of wrinkles, and forms locally suspended membranes over multiple holes in the substrate. (c) Schematic depiction of the indentation experiment. During measurements, the AFM tip is placed above the center and slowly lowered while monitoring its deflection.

cantilever (Olympus AC240). Figure 1c shows the schematic depiction of the experiment. For each hole, an AFM topographical image of the suspended membrane was acquired in amplitude modulation mode and used to position the tip in the middle of the membrane; see Figure 2a.

Cantilever deflection is measured while the probe is moved in the vertical direction with a speed of $2 \mu \mathrm{m}$ $\mathrm{s}^{-1}$, resulting in controlled loading and unloading of the suspended $\mathrm{MoS}_{2}$ membrane. Typical force versus piezo extension curves are shown on Figure $2 \mathrm{~d}$. Mechanical drift is minimized by means of a temperature controller integrated with the AFM system. Multiple curves with increasing indentation depths were acquired for each hole until mechanical failure was observed, as illustrated in Figure 2b. Mechanical failure typically occurs for vertical deflections $<50 \mathrm{~nm}$, well below the hole depth. No evidence of $\mathrm{MoS}_{2}$ sheets sliding over the substrate was observed. The height profile in Figure $2 \mathrm{c}$ shows that the membrane adheres to the sidewalls of the hole over a distance on the order of $5 \mathrm{~nm}$. This adhesion is due to van der Waals interaction and is presumably at the origin of the membrane pretensioning. Similar sidewall adhesion and pretension was reported in suspended graphene membranes. ${ }^{24,30}$ SEM imaging was used to check tip quality, confirming that no damage occurred to the $\mathrm{Si}$ AFM tips during measurements. This observation is also supported by the fact that AFM image quality and measured results did not show any observable change during the experiment. 


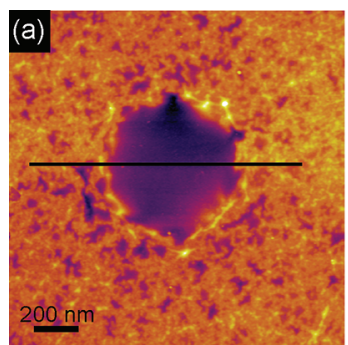

(c)

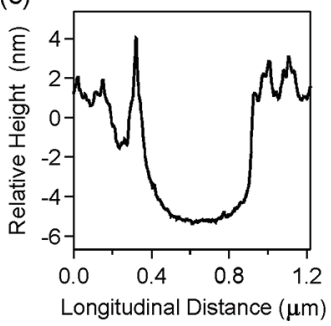

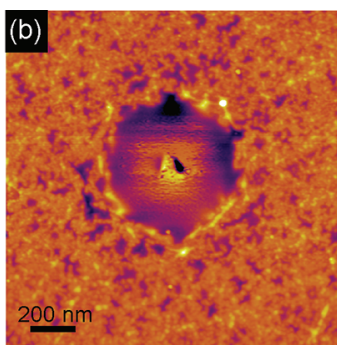

(d)

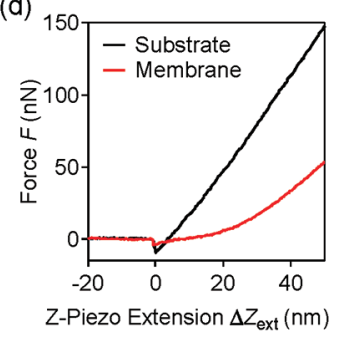

Figure 2. Suspended $\mathrm{MoS}_{2}$ membranes and their mechanical failure. (a) AFM image of a monolayer $\mathrm{MoS}_{2}$ flake suspended over a hole before the indentation experiment and (b) after it. A hole can be clearly seen in the center of the membrane at the location where the AFM tip punctured it. (c) Height profile of the section highlighted in (a) shows that the membrane adheres to the sidewalls over a vertical distance on the order of $5 \mathrm{~nm}$, resulting in pretension between 0.02 and $0.1 \mathrm{Nm}^{-1}$. (d) Acquired force versus z-piezo extension curves for the suspended membrane and the substrate.

We performed experiments on a total of 9 monolayer $(1 \mathrm{~L})$ and 6 double-layer $(2 \mathrm{~L}) \mathrm{MoS}_{2}$ membranes. A representative force $F$ versus z-piezo extension $\left(\Delta Z_{\text {ext }}\right)$ curve for a monolayer membrane and the substrate is shown on Figure $2 \mathrm{~d}$. Loading and unloading curves in general overlap, indicating that no plastic deformation or membrane detachment occurs during the measurements and that the material can be considered as elastic.

The schematic diagram of the measurement geometry is given in Figure 3a. Because the measurement geometry (both tip and the sample) has circular symmetry and $\mathrm{MoS}_{2}$ has six equivalent crystalline directions in terms of the stress-strain response, we model $\mathrm{MoS}_{2}$ as an isotropic film characterized by Young's modulus $E_{\mathrm{Y}}$, Poisson ratio $v$ and thickness $h$. Simulations on $\operatorname{MoS}_{2}$ and measurements on $\mathrm{WS}_{2}$ nanotubes ${ }^{18,19}$ show that these materials related to monolayer $\mathrm{MoS}_{2}$ are brittle and deform as materials with linear stress-strain relationship up to their failure. This is in stark contrast to carbon nanotubes or graphene where carbon atom chain formation and Stone-Wales transformations can lead to ductile behavior. ${ }^{31}$ Such mechanisms are absent in $\mathrm{MoS}_{2} \cdot{ }^{19}$ Because $\mathrm{MoS}_{2}$ monolayers and nanotubes share the same type of chemical bonds, we also model $\mathrm{MoS}_{2}$ as a linear elastic material.

The membrane is suspended over a circular hole with diameter $a=2 r=550 \pm 10 \mathrm{~nm}$ and deformed in the middle by an AFM tip with a radius $r_{\text {tip }}=12 \pm 2 \mathrm{~nm}$. We suppose that the film is prestretched due to van der Waals adhesion between the film and the substrate leading to internal strain $\varepsilon_{0}$. Mechanical response of

(a)

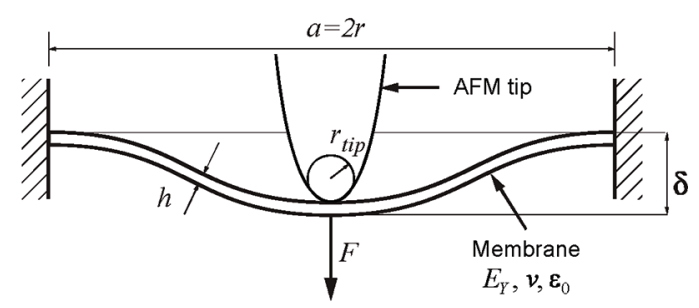

(b)

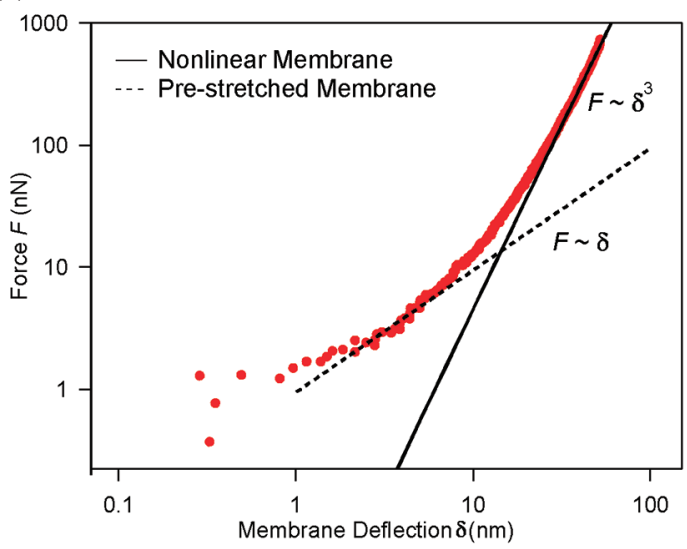

(c)

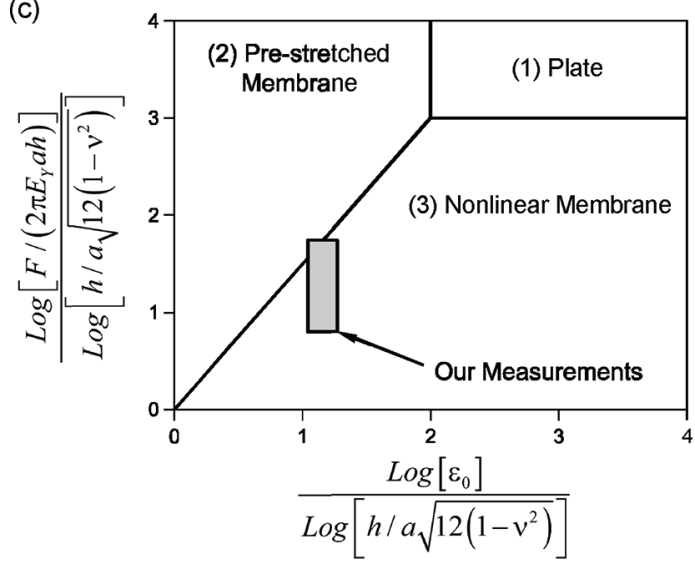

Figure 3. (a) Schematic diagram of the indentation test. A circular suspended membrane with Young's modulus $E_{\mathrm{Y}}$, Poisson ratio $v$, and prestrain $\varepsilon_{0}$ is elastically deformed in the middle by an AFM tip. The membrane is clamped at the edges and is loaded in the middle, resulting in membrane deflection $\delta$. (b) Typical loading curve for a monolayer $\mathrm{MoS}_{2}$ membrane. Small loads are characterized by a linear relationship between load $F$ and deflection $\delta$. For high loads, a cubic $F \sim \delta^{3}$ behavior dominates. (c) Parameter space according to Komaragiri et $a l^{32}$ delineating between different regimes of mechanical behavior for suspended circular membranes. The region in which our measurements are performed is shaded in gray.

such free-standing films can then fall into three distinct regimes, depending on the geometric factor $h / a$, applied load, and internal prestrain $\varepsilon_{0}{ }^{32}$ Linear plate bending and prestrained membrane deflection are characterized by linear force versus deflection behavior and are valid for small loads. Which one of these two governs the mechanical behavior depends on whether stiffness generated by prestrain is greater than the bending stiffness. The nonlinear membrane behavior 
characterized by a cubic $F \sim \delta^{3}$ relationship dominates at large loads. In order to elucidate between these different deformation regimes, we plot a typical forcedeflection curve in a logarithmic scale on Figure 3b. We can see that for small deflection and loading forces, limited by the resolution of our setup to $\sim 1 \mathrm{nN}$, the curve follows a linear behavior (dashed line). For forces $>10 \mathrm{nN}$, the curve starts to follow cubic behavior, typical of deformed membranes. We therefore fit the force-deflection data using a formula that captures both the linear behavior for small deformations and the cubic term for larger deformations: ${ }^{32}$

$$
F=\sigma_{0}^{2 \mathrm{D}} \pi \delta+E^{2 \mathrm{D}} \frac{q^{3} \delta^{3}}{r^{2}}
$$

where the cubic term containing the elastic modulus $E^{2 \mathrm{D}}$ represents the modified form of the classical Schwerin solution for point loading of a circular membrane ${ }^{33}$ valid for all values of the Poisson's ratio. ${ }^{32}$ The term linear with deflection $\delta$, where $\sigma_{0}^{2 \mathrm{D}}$ is the prestress in the membrane, corresponds to the linear,

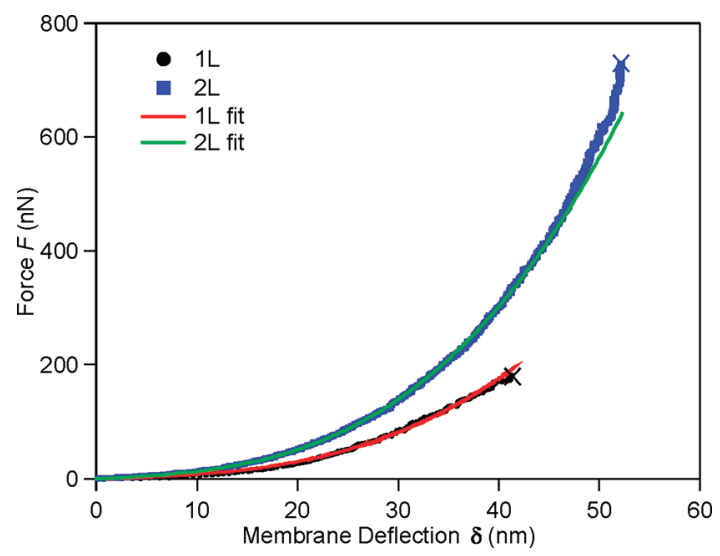

Figure 4. Examples of loading curves for single and bilayer $\mathrm{MoS}_{2}$ and the least-squares fit of the experimental indentation curves to the eq 1 . The fitting allows us to extract the pretension of the membrane $\sigma_{0}^{2 \mathrm{D}}$ and its Young modulus $E^{2 \mathrm{D}}$. Experimental and fitted curves show good agreement. Membranes are fractured at the point marked by the symbol $\times$.

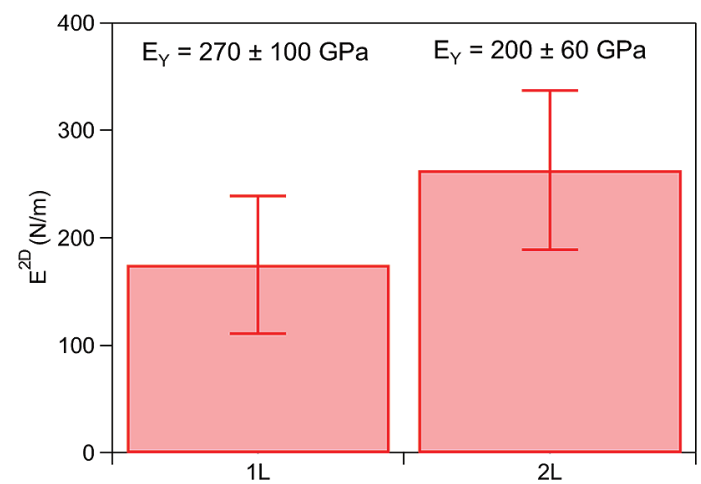

prestretched membrane regime with an internal strain of $\varepsilon_{0}$.

Finite element simulations by Lee et al. ${ }^{24}$ showed that this model can be applied to small and finite sized indenters as long as $r_{\text {tip }} \ll r$. We extract the tip radius $r_{\text {tip }}$ $\sim 12 \mathrm{~nm}$ from SEM images, resulting in $r_{\text {tip }} / r=0.05$ in our case. The dimensionless constant $q$ is related to the Poisson ratio $v$ as $q=1 /\left(1.05-0.15 v-0.16 v^{2}\right)=0.95$, where $v=0.27$ is the Poisson ratio of bulk $\mathrm{MoS}_{2}{ }^{34}$ The map of possible regimes of film response according to Komaragiri et al. ${ }^{32}$ is presented on Figure 3c, together with the range of range of tests covered in this paper, confirming that most of the mechanical response that we record is expected to be in the region described by the cubic $F \sim \delta^{3}$ behavior.

Figure 4 shows representative experimental forcedeflection curves acquired for mono- and bilayer $\mathrm{MoS}_{2}$. From a least-squares fit of the experimental curves with the eq 1 , we can extract the pretension $\sigma_{0}^{2 \mathrm{D}}$ and the membrane elastic modulus $E^{2 \mathrm{D}}$. The fit agrees well with experimental data, validating our assumptions that led to the choice of eq 1 as the model. For a total of 9 monolayers $(1 \mathrm{~L})$, we obtain the average value for the elastic modulus $E^{2 \mathrm{D}}$ of $180 \pm 60 \mathrm{Nm}^{-1}$ (Figure 5, left) and prestress $\sigma_{0}^{2 \mathrm{D}}$ in the 0.02 to $0.1 \mathrm{Nm}^{-1}$ range. Throughout the paper, the experimental uncertainty for the measured values of the elastic modulus and the breaking strength corresponds to the standard deviation of experimental values. Assuming an effective monolayer thickness of $0.65 \mathrm{~nm}$, we obtain for the Young's modulus $E_{\text {Young }}=270 \pm 100 \mathrm{GPa}$, close to the Young's modulus of $\mathrm{MoS}_{2}$ nanotubes (230 GPa, ref 18), bulk $\mathrm{MoS}_{2}$ (238 GPa, ref 34), or steel (210 GPa, ref 35). The elastic modulus of bilayer $\mathrm{MoS}_{2}$ is $260 \pm$ $70 \mathrm{Nm}^{-1}$, which corresponds to a lower Young's modulus of $200 \pm 60 \mathrm{GPa}$, possibly due to defects or interlayer sliding.

During nanomechanical measurements, suspended membranes are deformed up to their mechanical failure, denoted by the symbol $x$ in Figure 4, typically occurring for fracture forces of $F_{\max } \approx 200 \mathrm{nN}$ and

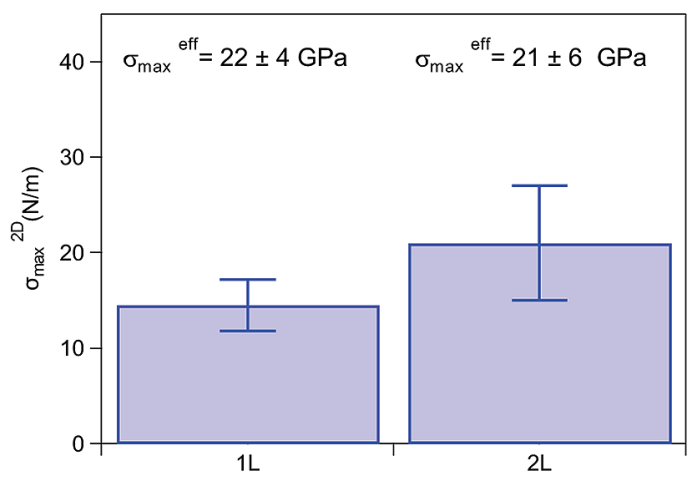

Figure 5. (Left) Young's modulus $E^{2 \mathrm{D}}$ and (right) maximum breaking stress $\sigma_{\max }^{2 \mathrm{D}}$ at the central point of the film for $1 \mathrm{~L}$ and $2 \mathrm{~L}$ $\mathrm{MoS}_{2}$ flakes extracted from the experimental data. The corresponding effective bulk modulus assuming a monolayer flake thickness of $6.5 \AA$ is also shown for comparison with the bulk material. 
TABLE 1. Comparison of Young's Moduli and Breaking Strengths for Several Engineering Materials, Including Monolayer $\operatorname{MoS}_{2}{ }^{19,24,34,35,39-43}$

\begin{tabular}{|c|c|c|c|}
\hline material & $\begin{array}{l}\text { Young's modulus } \\
E_{\text {Young }}(\mathrm{GPa})\end{array}$ & $\begin{array}{l}\text { breaking strength } \\
\sigma_{\max }^{\text {eff }}(\mathrm{GPa})\end{array}$ & $\begin{array}{c}\text { breaking } \\
\text { strength/Young's } \\
\text { modulus (\%) }\end{array}$ \\
\hline $\begin{array}{l}\text { stainless steel } \\
\text { ASTM-A514 }\end{array}$ & 205 & 0.9 & 0.4 \\
\hline molybdenum & 329 & $0.5-1.2$ & $0.15-0.36$ \\
\hline polyimide & 2.5 & 0.231 & 9 \\
\hline PDMS & $0.3-0.87$ & 2.24 & 2.5 \\
\hline Kevlar 49 & 112 & 3 & 2.6 \\
\hline monolayer $\mathrm{MoS}_{2}$ & 270 & $16-30$ & $6-11$ \\
\hline bulk $\mathrm{MoS}_{2}$ & 238 & & \\
\hline$W S_{2}$ nanotubes & 152 & $3.7-16.3$ & $2.4-10$ \\
\hline carbon nanotubes & 1000 & $11-63$ & $1.1-6.3$ \\
\hline graphene & 1000 & 130 & 13 \\
\hline
\end{tabular}

deflections $\approx 50 \mathrm{~nm}$. For such extreme deformation, in addition to large-scale deformation, the membrane is locally indented within a typical area extending $\sim 2 r_{\text {tip }}$ distance from the center. ${ }^{32}$ Here, the local curvature of the membrane is constrained by the tip diameter of the AFM probe, as long as the membrane is allowed to deform smoothly onto the indenter. Our experiment falls in the limit of small indenters characterized by $r_{\text {tip }} / r \ll 1\left(r_{\text {tip }} / r=0.05\right.$ in our case) and large loads with respect to pretension, characterized by a factor $\kappa \ll 1$ defined $\mathrm{as}^{36}$

$$
\kappa=\left(\frac{\sigma_{0}}{E_{Y}}\right)^{1 / 3}\left(\frac{2 r_{\text {tip }}}{r}\right)^{2 / 3}
$$

In our case, $\kappa<0.02$. Here, we can extract the maximum stress at the central, protruding part of the film $\sigma_{\max }^{2 D}$ using the expression for the indentation of a linearly elastic membrane by a spherical indenter in the limit of large load: ${ }^{36}$

$$
\sigma_{\max }^{2 \mathrm{D}}=\sqrt{\frac{F_{\max } E^{2 \mathrm{D}}}{4 \pi r_{\text {tip }}}}
$$

The averages of maximum stress values for $1 \mathrm{~L}$ and 2

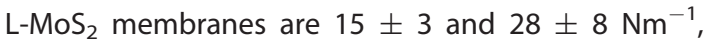
respectively (corresponding to $22 \pm 4 \mathrm{GPa}$ for a monolayer and $21 \pm 6 \mathrm{GPa}$ for a bilayer), as reported in Figure 4. On the average, these correspond to 8 and $10 \%$ of the Young's modulus for monolayer and bilayer $\mathrm{MoS}_{2}$. The strength of individual $\mathrm{MoS}_{2}$ monolayers is between 6 and $11 \%$ of their Young's modulus. These values are at the theoretical upper limit of a material's breaking strength and thus represent the intrinsic strength of interatomic bonds in $\mathrm{MoS}_{2}$. This exceptionally high strength indicates that $\mathrm{MoS}_{2}$ membranes are mostly defect-free. To put these high values of breaking strength and Young's modulus in perspective, we can compare them to several common engineering materials in Table 1. The strength of monolayer $\mathrm{MoS}_{2}$ is exceeded only by carbon nanotubes and graphene. Even though $\mathrm{MoS}_{2}$ has a smaller Young's modulus and strength than graphene, the absolute 2D elastic modulus $E^{2 \mathrm{D}}$ and strength $\sigma_{\max }^{2 \mathrm{D}}$ of monolayer $\mathrm{MoS}_{2}$ are smaller than those of graphene only by a factor of $\sim 2$.

In order to investigate if $\mathrm{MoS}_{2}$ could be suitable for integration with flexible materials, for example, in flexible electronic circuits, it is necessary to quantify the membrane strain at the breaking point, $\varepsilon_{\text {int }}$ and compare it to the breaking strain of standard flexible substrates. Assuming a linear relationship between stress and strain $\sigma=E \varepsilon$ leads to an internal stress at failure $\varepsilon_{\text {int }} \sim 0.06-0.11$.

In comparison, thin polymer films such as polyimide (PI) or polydimethylsiloxane (PDMS), commonly used as substrates for flexible electronics, break at a strain of $\sim 7 \%$ (ref 37), which is smaller than the aforementioned value extracted for single-layer $\mathrm{MoS}_{2}$. This suggests that $2 \mathrm{D} \mathrm{MoS}$ can be readily integrated with PI or PDMS substrates for use in flexible electronics.

\section{CONCLUSIONS}

Our results show that monolayer $\mathrm{MoS}_{2}$ is a flexible and strong material with a high Young's modulus, comparable to stainless steel. The measured strength of monolayer $\mathrm{MoS}_{2}$ is close to the theoretical intrinsic strength of the Mo-S chemical bond, indicating that the monolayer is mostly free of defects and dislocations capable of reducing mechanical strength. As $\mathrm{MoS}_{2}$ can be readily dispersed in a wide variety of solvents, ${ }^{9}$ our finding indicates that $\mathrm{MoS}_{2}$ could be interesting as a reinforcing element in composites. The presence of sulfur in $\mathrm{MoS}_{2}$ could furthermore allow easy functionalization and efficient load transfer between $\mathrm{MoS}_{2}$ and the composite matrix.

We also find that the exceptional mechanical properties of monolayer $\mathrm{MoS}_{2}$ make it suitable for incorporation into flexible electronic devices where commonly used substrates such a PI would undergo mechanical failure at a smaller deformation than $\operatorname{MoS}_{2}$.

\section{MATERIALS AND METHODS}

Single layers of $\mathrm{MoS}_{2}$ are exfoliated from commercially available crystals of molybdenite (SPI Supplies Brand Moly Disulfide) using the scotch-tape micromechanical cleavage technique pioneered for the production of graphene. ${ }^{1}$ Monolayer and few-layer $\mathrm{MoS}_{2}$ was first deposited on a silicon substrate with $270 \mathrm{~nm}$ thick $\mathrm{SiO}_{2}$ that has previously been coated with polyvinyl alcohol (Sigma-Aldrich) and polymethyl methacrylate (PMMA, Microchem Corp). The PMMA film is released by dissolving PVA in water and transferred on top of a prepatterned $\mathrm{SiO}_{2}$ substrate. After transfer, PMMA is removed by heating the 
sample in a vacuum furnace at $400^{\circ} \mathrm{C}$ for $4 \mathrm{~h}$. AFM imaging and indentation experiment was performed using the Asylum Research Cypher AFM equipped with the air temperature controller for minimizing drift and using Olympus AC240 silicon cantilevers. AFM probe spring constants were calibrated using the thermal method. ${ }^{38}$

Acknowledgment. Substrate fabrication was carried out in part in the EPFL Center for Micro/Nanotechnology (CMI). We thank Michael Brian Whitwick for help with SEM imaging of AFM tips. This work was financially supported the Swiss National Science Foundation (Grant No. 200021_132102) and the Swiss Nanoscience Institute (NCCR Nanoscience).

Supporting Information Available: SEM images of atomic force microscope probes. This material is available free of charge via the Internet at http://pubs.acs.org.

\section{REFERENCES AND NOTES}

1. Novoselov, K. S.; Geim, A. K.; Morozov, S. V.; Jiang, D.; Zhang, Y.; Dubonos, S. V.; Grigorieva, I. V.; Firsov, A. A. Electric Field Effect in Atomically Thin Carbon Films. Science 2004, 306, 666-669.

2. Frindt, R. F. Single Crystals of $\mathrm{MoS}_{2}$ Several Molecular Layers Thick. J. Appl. Phys. 1966, 37, 1928-1929.

3. Novoselov, K. S.; Jiang, D.; Schedin, F.;Booth, T. J.; Khotkevich, V. V.; Morozov, S. V.; Geim, A. K. Two-Dimensional Atomic Crystals. Proc. Natl. Acad. Sci. U.S.A. 2005, 102, 10451-10453.

4. Novoselov, K. S.; Geim, A. K.; Morozov, S. V.; Jiang, D.; Katsnelson, M. I.; Grigorieva, I. V.; Dubonos, S. V.; Firsov, A. A. Two-Dimensional Gas of Massless Dirac Fermions in Graphene. Nature 2005, 438, 197-200.

5. Zhang, Y.; Tan, Y.-W.; Stormer, H. L.; Kim, P. Experimental Observation of the Quantum Hall Effect and Berry's Phase in Graphene. Nature 2005, 438, 201-204.

6. Du, X.; Skachko, I.; Duerr, F.; Luican, A.; Andrei, E. Y. Fractional Quantum Hall Effect and Insulating Phase of Dirac Electrons in Graphene. Nature 2009, 462, 192-195.

7. Joensen, P.; Frindt, R. F.; Morrison, S. R. Single-Layer $\mathrm{MoS}_{2}$. Mater. Res. Bull. 1986, 21, 457-461.

8. Schumacher, A.; Scandella, L.; Kruse, N.; Prins, R. SingleLayer $\mathrm{MoS}_{2}$ on Mica: Studies by Means of Scanning Force Microscopy. Surf. Sci. Lett. 1993, 289, L595-L598.

9. Coleman, J. N.; Lotya, M.; O'Neill, A.; Bergin, S. D.; King, P. J.; Khan, U.; Young, K.; Gaucher, A.; De, S.; Smith, R. J.; et al. Two-Dimensional Nanosheets Produced by Liquid Exfoliation of Layered Materials. Science 2011, 331, 568-571.

10. Radisavljevic, B.; Radenovic, A.; Brivio, J.; Giacometti, V.; Kis, A. Single-Layer $\mathrm{MoS}_{2}$ Transistors. Nat. Nanotechnol. 2011, 6, 147-150.

11. Kam, K. K.; Parkinson, B. A. Detailed Photocurrent Spectroscopy of the Semiconducting Group Vib Transition Metal Dichalcogenides. J. Phys. Chem. 1982, 86, 463-467.

12. Lebegue, S.; Eriksson, O. Electronic Structure of TwoDimensional Crystals from $A b$ Initio Theory. Phys. Rev. $B$ 2009, 79, 115409 .

13. Splendiani, A.; Sun, L.; Zhang, Y.; Li, T.; Kim, J.; Chim, C.-Y.; Galli, G.; Wang, F. Emerging Photoluminescence in Monolayer $\mathrm{MoS}_{2}$. Nano Lett. 2010, 10, 1271-1275.

14. Mak, K. F.; Lee, C.; Hone, J.; Shan, J.; Heinz, T. F. Atomically Thin $\mathrm{MoS}_{2}$ : A New Direct-Gap Semiconductor. Phys. Rev. Lett. 2010, 105, 136805.

15. Kuc, A.; Zibouche, N.; Heine, T. Influence of Quantum Confinement on the Electronic Structure of the Transition Metal Sulfide TS 2 . Phys. Rev. B 2011, 83, 245213.

16. Tenne, R.; Margulis, L.; Genut, M.; Hodes, G. Polyhedral and Cylindrical Structures of Tungsten Disulfide. Nature 1992, 360, 444-446.

17. Tenne, R.; Homyonfer, M.; Feldman, Y. Nanoparticles of Layered Compounds with Hollow Cage Structures (Inorganic Fullerene-like Structures). Chem. Mater. 1998, 10,3225 .
18. Kaplan-Ashiri, I.; Cohen, S. R.; Gartsman, K.; Rosentsveig, R.; Seifert, G.; Tenne, R. Mechanical Behavior of Individual WS Nanotubes. J. Mater. Res. 2004, 19, 454-459.

19. Kaplan-Ashiri, I.; Cohen, S. R.; Gartsman, K.; Ivanovskaya, V.; Heine, T.; Seifert, G.; Wiesel, I.; Wagner, H. D.; Tenne, R. On the Mechanical Behavior of WS $_{2}$ Nanotubes under Axial Tension and Compression. Proc. Natl. Acad. Sci. U.S.A. 2006, 103, 523-528.

20. Kis, A.; Mihailovic, D.; Remskar, M.; Mrzel, A.; Jesih, A.; Piwonski, I.; Kulik, A. J.; Benoit, W.; Forró, L. Shear and Young's Moduli of $\mathrm{MoS}_{2}$ Nanotube Ropes. Adv. Mater. 2003, 15, 733-736.

21. Griffith, A. The Phenomenon of Rupture and Flow in Solids. Philos. Trans. $R$ Soc. 1920, 221, 163.

22. Frank, I. W.; Tanenbaum, D. M.; van der Zande, A. M.; McEuen, P. L. Mechanical Properties of Suspended Graphene Sheets. J. Vac. Sci. Technol., B 2007, 25, 2558-2561.

23. Poot, M.; van der Zant, H. S. J. Nanomechanical Properties of Few-Layer Graphene Membranes. Appl. Phys. Lett. 2008, 92, 063111.

24. Lee, C.; Wei, X.; Kysar, J. W.; Hone, J. Measurement of the Elastic Properties and Intrinsic Strength of Monolayer Graphene. Science 2008, 321, 385-388.

25. Mohiuddin, T. M. G.; Lombardo, A.; Nair, R. R.; Bonetti, A.; Savini, G.; Jalil, R.; Bonini, N.; Basko, D. M.; Galiotis, C.; Marzari, N.; et al. Uniaxial Strain in Graphene by Raman Spectroscopy: G Peak Splitting, Grüneisen Parameters, and Sample Orientation. Phys. Rev. B 2009, 79, 205433.

26. Tsoukleri, G.; Parthenios, J.; Papagelis, K.; Jalil, R.; Ferrari, A. C.; Geim, A. K.; Novoselov, K. S.; Galiotis, C. Subjecting a Graphene Monolayer to Tension and Compression. Small 2009, 5, 2397-2402.

27. Dean, C. R.; Young, A. F.; Cadden-Zimansky, P.; Wang, L.; Ren, H.; Watanabe, K.; Taniguchi, T.; Kim, P.; Hone, J.; Shepard, K. L. Multicomponent Fractional Quantum Hall Effect in Graphene. Nat. Phys. 2011, 7, 693-696.

28. Benameur, M. M.; Radisavljevic, B.; Heron, J. S.; Sahoo, S.; Berger, $\mathrm{H}_{\text {.; }}$ Kis, A. Visibility of Dichalcogenide Nanolayers. Nanotechnology 2011, 22, 125706.

29. Weisenhorn, A. L.; Hansma, P. K.; Albrecht, T. R.; Quate, C. F. Forces in Atomic Force Microscopy in Air and Water. Appl. Phys. Lett. 1989, 54, 2651-2653.

30. Bunch, J. S.; Verbridge, S. S.; Alden, J. S.; van der Zande, A. M.; Parpia, J. M.; Craighead, H. G.; McEuen, P. L. Impermeable Atomic Membranes from Graphene Sheets. Nano Lett. 2008, 8, 2458-2462.

31. Nardelli, M. B.; Yakobson, B. I.; Bernholc, J. Brittle and Ductile Behavior in Carbon Nanotubes. Phys. Rev. Lett. 1998, 81, 4656-4659.

32. Komaragiri, U.; Begley, M. R.; Simmonds, J. G. The Mechanical Response of Freestanding Circular Elastic Films under Point and Pressure Loads. J. Appl. Mech. 2005, 72, 203-212.

33. Schwerin, E. Uber Spannungen Und Formänderungen Kreisringförmiger Membranen. Z. Tech. Phys. 1929, 12, 651-659.

34. Feldman, J. L. Elastic Constants of $2 \mathrm{~h}-\mathrm{MoS}_{2}$ and $2 \mathrm{~h}-\mathrm{NbSe}_{2}$ Extracted from Measured Dispersion Curves and Linear Compressibilities. J. Phys. Chem. Solids 1976, 37, 11411144.

35. Gere, J. M.; Timoshenko, S. P., Mechanics of Materials, 3rd ed.; PWS-Kent: Boston, MA, 1984; p 690.

36. Bhatia, N. M.; Nachbar, W. Finite Indentation of an Elastic Membrane by a Spherical Indenter. Int. J. Non-Linear Mech. 1968, 3, 307-324.

37. Kim, D.-H.; Ahn, J.-H.; Choi, W. M.; Kim, H.-S.; Kim, T.-H.; Song, J.; Huang, Y. Y.; Liu, Z.; Lu, C.; Rogers, J. A. Stretchable and Foldable Silicon Integrated Circuits. Science 2008, 320, 507-511.

38. Hutter, J. L.; Bechhoefer, J. Calibration of Atomic-Force Microscope Tips. Rev. Sci. Instrum. 1993, 64, 1868-1873.

39. Shinno, H.; Kitajima, M.; Okada, M. Thermal Stress Analysis of High Heat Flux Materials. J. Nucl. Mater. 1988, 155-157, 290-294.

40. Dupont Kapton Polyimide Film General Specifications, Bulletin Gs-96-7. 
41. Armani, D.; Liu, C.; Aluru, N. In Re-Configurable Fluid Circuits by Pdms Elastomer Micromachining, Micro Electro Mechanical Systems, 1999. MEMS '99; 12th IEEE International Conference, 17-21 Jan, 1999; pp 222-227.

42. Kevlar Technical Guide.

43. Yu, M.-F.; Lourie, O.; Dyer, M. J.; Moloni, K.; Kelly, T. F.; Ruoff, R. S. Strength and Breaking Mechanism of Multiwalled Carbon Nanotubes under Tensile Load. Science 2000, 287, 637-640. 